Available online at $\quad$ http://www.jfas.info

\title{
INTERACTIVE LEARNING SOFTWARE FOR ELECTRICAL ENGINEERING SUBJECTS USING MATLAB AND ITS GUI
}

\author{
N. F. Naim*, H. I. M. Zaini, S. S. Sarnin and N. Ya'acob \\ Faculty of Electrical Engineering, UniversitiTeknologi MARA, 40450 Shah Alam, Selangor, \\ Malaysia
}

Published online: 17 October 2017

\begin{abstract}
This paper presents a user-friendly learning software using MATLAB and its Graphical User Interface (GUI) to help student in achieving a better understanding in electrical engineering subjects. Currently, this educational software is designed for three subjects; electromagnetic, digital communication and power engineering. The $\mathrm{m}$-files are developed and their graphical user interfaces (GUIs) are designed for ease of use. The standalone program is also developed; thus, user does not need to install MATLAB in their Personal Computer (PC) in order to use this educational software.
\end{abstract}

Keywords: electrical engineering; MATLAB; graphic user interface (GUI); educational software.

Author Correspondence, e-mail: nanifadzlina@salam.uitm.edu.my doi: http://dx.doi.org/10.4314/jfas.v9i5s.19 


\section{INTRODUCTION}

MATLAB which stands for Matrix Laboratory, is a high performance numerical analysis and calculation software introduced by Mathworks Company based in the USA. The researcher in [1-3] stated that MATLAB has unique advantages in the matrix algebra, numerical computing, digital signal processing, system identification, dynamic system simulation, graphic processing and many other areas.

There have been several studies that have employed GUI of MATLAB to help them to obtain the result in the form of graphical images such as in [3-4] studied on Self Excited Induction Generator (SEIG). The study involved the energy conversion device to produce electricity in off-grid, stand-alone mode using different types of prime movers and employing different conventional and renewable energy. The analysis of such experiment requires a suitable user friendly tool to predict the performance under different operating conditions to facilitate proper design of the system. The researchers had developed analytical design tool and software using MATLAB tool boxes such as "Fsolve" routine and the GUI can effectively use to predict the performance of SEIG with different types of prime movers.

Meanwhile in [5], the researchers used MATLAB and its GUI in order to tackle the problem of dynamics of electric machines through simulation of starting of an asynchronous machine by producing a computer-generated laboratory. This paper discussed the asynchronous motor of a fan used in auxiliary's posts of electromotive. The researchers wanted to create a virtual laboratory which can be a space where elements were expressed virtually using interactive graphics and design. The computational modelling of the induction machine had offered more information and eases their working operation in diverse situation.

\section{DESIGN PRINCIPLE}

In order to develop this educational software, several studies have been done. All of the equations are developed using MATLAB codes and saved in the form of $m$-files. Then, the GUI is designed carefully to ensure that it can be easily used by the user.

\subsection{Development of M-Files for Electromagnetic Coordinate System}

With the aim of describing the spatial variations of the function of space and time, all the points in space must be defined specifically using a suitable coordinate system [6-9]. The 
most important and useful coordinate systems are:

i) Cartesian, or Rectangular coordinates.

ii) Cylindrical or Circular coordinates.

iii) Spherical or Polar coordinates.

Thus, the system has been developed with capability of converting the values in any of the coordinate systems required.

\subsection{Development of M-Files for Producing Dot Product, Cross Product and Electric Field}

\section{Intensity}

Cross product can be defined as the interaction of vectors in different dimensions. Dot product is the interaction between vectors in the same dimensions. On the other hand, electric field intensity $\vec{E}$ is the strength of an electric field at any point. Then, the intensity of electric field at source point $Q$ at location $\overrightarrow{r_{1}}$ due to a point observation of $P$ at location $\overrightarrow{r_{2}}$ is:

$$
\vec{E}=\frac{k Q_{\text {source }}}{R^{2}}=\frac{Q_{\text {source }}\left(r_{2}-\overrightarrow{r_{1}}\right)}{4 \pi \varepsilon_{0}\left|\overrightarrow{r_{2}-r_{1}}\right|^{3}}
$$

\subsection{Development of M-Files for High Frequency (HF) Analysis and Propagation of Electromagnetic Wave}

The graphical simulation of electromagnetic wave in free space and good conductor are demonstrated, according to the equation below as obtained from (6):

$$
\vec{\nabla}^{2} \vec{A}_{s}-\gamma^{2} \vec{A}_{s}=0
$$

where $\vec{A}_{s}$ is either $\vec{E}_{s}$ or $\vec{H}_{s}$ and $\gamma=\alpha+j \beta$ is the propagation constant. Assuming $\overrightarrow{E_{s}}=E_{x s}(z) \overrightarrow{u_{x}}$, electromagnetic waves of the form can be obtained as follows:

$$
\begin{array}{r}
\vec{E}(z, t)=E_{0} e^{-a z} \cos (\omega t-\beta z) \overrightarrow{u_{x}} \\
\vec{H}(z, t)=H_{0} e^{-a z} \cos \left(\omega t-\beta z-\theta_{\eta}\right) \vec{u}_{y}
\end{array}
$$

where $\alpha=$ attenuation constant, $\beta=\frac{2 \pi}{\lambda}=$ phase constant, $\eta=$ the medium's intrinsic 
impedance and $\theta_{\eta}=$ phase difference between $\vec{E}$ and $\vec{H}$.

Moving on to the next section is the High Frequency (HF) analysis part, the process concentrated on the graphical user interface (GUI). The main purpose of this program is to analyze the high frequency signal where users can choose to study Amplitude-Shift Keying, Frequency-Shift Keying, multi Amplitude-Shift Keying or Morse Code. All of this analysis will be done in offline mode.

\subsection{Development of $m$-files for Digital Modulation subject}

For digital modulation which is located under the Digital Communication section of this project, an additional sub topics are included which is the Phase-Shift Keying (PSK). The basic PSK learned by Communication Engineering students are the Binary Phase-Shift Keying (BPSK) and Quadrature Phase-Shift Keying (QPSK). Therefore, these two sub topics are added into the digital modulation topic. The previous version of learning software in [10] has included the Amplitude-Shift Keying (ASK) and Frequency-Shit Keying (FSK) under the digital modulation topic. With the presents of BPSK and QPSK, students can look more into digital modulation and hopefully be able to differentiate between the digital modulations better.

\subsubsection{Binary Phase-Shift Keying (BPSK)}

The simplest form of PSK is the Binary Phase Shift Keying (BPSK) which uses two phases that are separated by $180^{\circ}$. This is why BPSK also can be known as 2-PSK or Phase Reversal Keying [11]. This modulation is the most vigorous compared to other PSKs that can cause the demodulator reach an incorrect decision as the noise produced is very high. However, it is only able to modulate $1 \mathrm{bit} / \mathrm{symbol}$. Therefore, it is incompatible for applications that require a high data-rate. The common equation for BPSK is

$$
s_{n}(t)=A \cos \left(2 \pi f_{c} t+\pi(1-n)\right), n=0,1
$$

\subsubsection{Quadrature Phase-Shift Keying (QPSK)}

Quadrature Phase-Shift Keying (QPSK) or also known as Quadriphase PSK or 4-PSK, uses four points on constellation points that surround a circle [11]. In these four phases, QPSK can minimize the bit error rate (BER) by encoding two bit per symbol. Mathematically, QPSK is proven to be either produce twice the data rate of BPSK but preserve the same bandwidth of 
the signal, or keep the data rate of BPSK but go halves on the bandwidth needed. In the later cases, the BER of QPSK will be exactly the same as the BER of BPSK. In QPSK, two sinusoids ( $\sin$ and cos) are taken as basis function for modulation. The following equation shows the QPSK modulation technique.

$$
s_{n}(t)=A \cos \left(2 \pi f_{c} t+(2 n-1) \frac{\pi}{4}\right), n=1,2,3,4
$$

As mentioned before, QPSK produced four sine signal shifted by $45^{\circ}$ from one another. The two phases are added together to produce the final signal where each unique pair of bits generates a carrier with different phase.

\subsection{Development of M-Files for Power Engineering Subject}

The main focus of this subject is to ensure that students can understand the basic principles of power systems and electrical machines [12]. One of the important topics learned by students is the Balanced 3Phase Systems. In this topic, students have to learn about the existence of star and delta connected load (resistor) and ways to convert it.

\subsubsection{Star to Delta Resistive Conversion}

Star connected network holds the symbol of letter, Y (wye) while Delta connected network holds the symbol of a triangle, $\Delta$ (delta). Besides, a form of Star or Y network type in a configuration is equivalent to the $\mathrm{T}$ or "Tee" form of configuration [12]. As for the transformation from star to delta configuration, can be seen using the following Fig. 1.

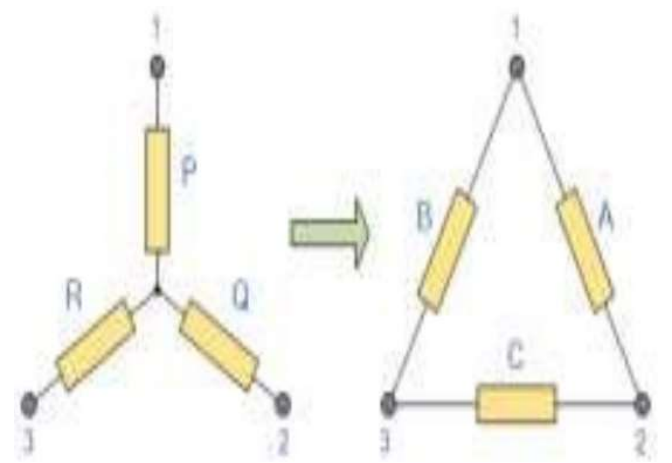

Fig.1. Guide in transforming star to delta connection

The following calculations are:

$$
\begin{aligned}
& A=\frac{P \times Q}{R}+Q+P \\
& B=\frac{R \times P}{Q}+P+R
\end{aligned}
$$




$$
C=\frac{Q \times R}{P}+Q+R
$$

\subsubsection{Delta to Star Resistive Conversion}

While Star connection circuit can be equal to a T connected circuit, a Delta connected circuit can be equal to a Pi connected circuit [12]. As for the conversion from delta to star can refer to the following Fig. 2.

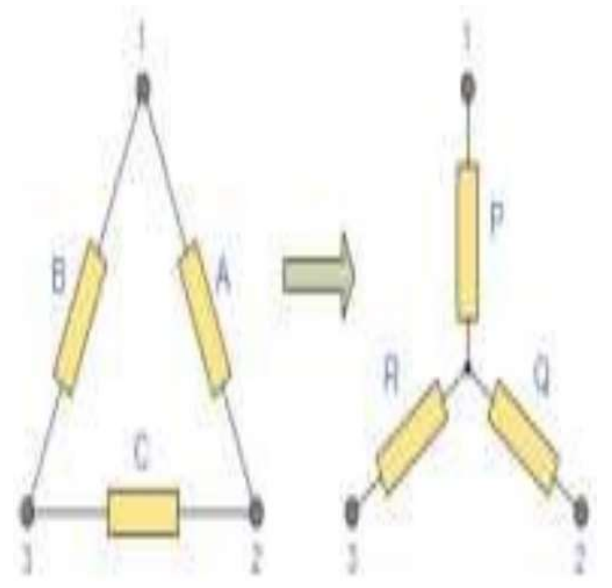

Fig.2. Guide to transform a delta to star connection

The calculations of following transformations are:

$$
\begin{aligned}
P=\frac{A \times B}{A+B+C}(10) & \\
Q & =\frac{A \times C}{A+B+C}(11) \\
R & =\frac{B \times C}{A+B+C}(12)
\end{aligned}
$$

\section{RESULTS AND DISCUSSION}

The software is designed using MATLAB R2013b environment. First is the starting system which is home system as shown in Fig. 3 consists of three options. The "Help" button can provide an aid to the user and user can depart from the program by clicking the "Exit" button. 
Thome?

UNIVERSITI TEKNOLOGI MARA MALAYSIA

Interactive Edtucational Software

Fox Engineering Subjects

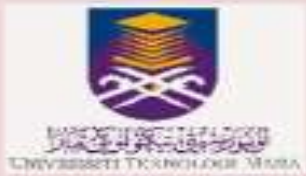

Flectromagnetic

Digital Communteation

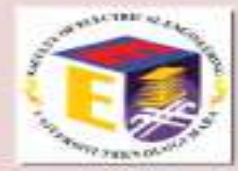

Power Engineering

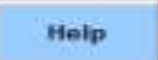

Designed ty: Hani Izzati Binti Md Zaini

Supervisor: Dr Nani Fadzlinat Binti Haim

Fig.3. Home of the system menu

On Electromagnetic mode as demonstrated in Fig. 4, there are six options to choose from, along with the "Home" button and the "Exit".

A) electromag

-

$\times$

\section{Electromagnetic}

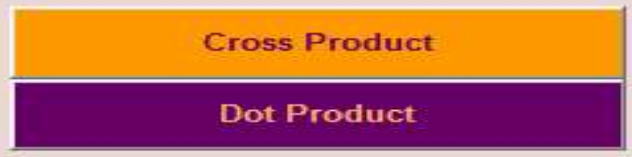

Electric Field Intensity due to a Charge

\section{Plotting Electric Field}

Electromagnetic Propagation

Coordinate System

Home

Exit

Fig.4. Electromagnetic mode

Fig. 5 shows the second main part which is the Digital Communication subject consist of two subtopics. In Fig. 6, "Binary Phase-Shift Keying”, "Quadrature Phase-Shift Keying" and "All Modulation" are other options added into the Digital Modulation mode. 


\section{Digital Communication}

Digital Modulation

High Frequency Analysis

Fig.5. Digital Communication mode

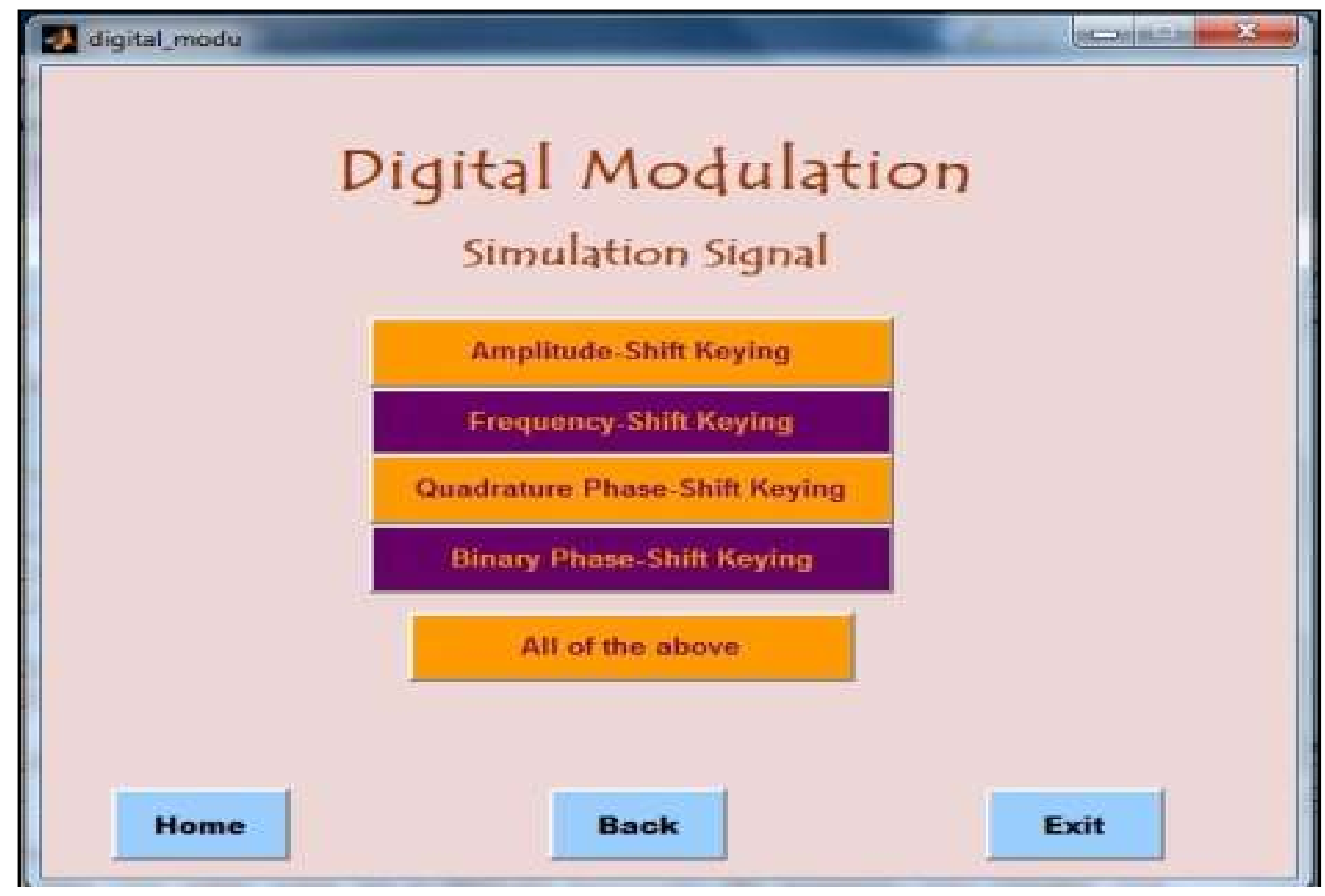

Fig.6. Digital Modulation mode

Lastly, in Fig. 7 shows the "Power Engineering" mode containing 3-phase resistive conversion of "Delta to Star" and "Star to Delta". 
3 Phase Circuit Converter (Resistive Connected)

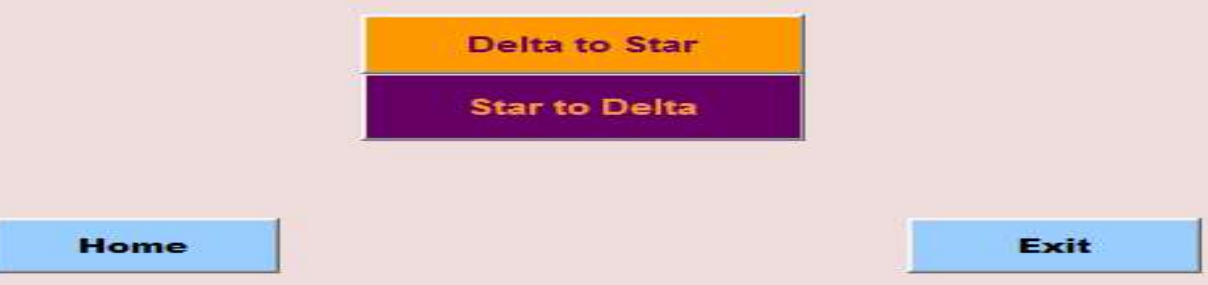

Fig.7. Power Engineering mode

These section is designed where user is required to enter the value of vector $a$ and $b$ according to the dimensions $x, y$ and $z$ severally. Then, the "Answer" button will provide the answer needed when it is clicked as shown in Fig. 8 for cross product's example.

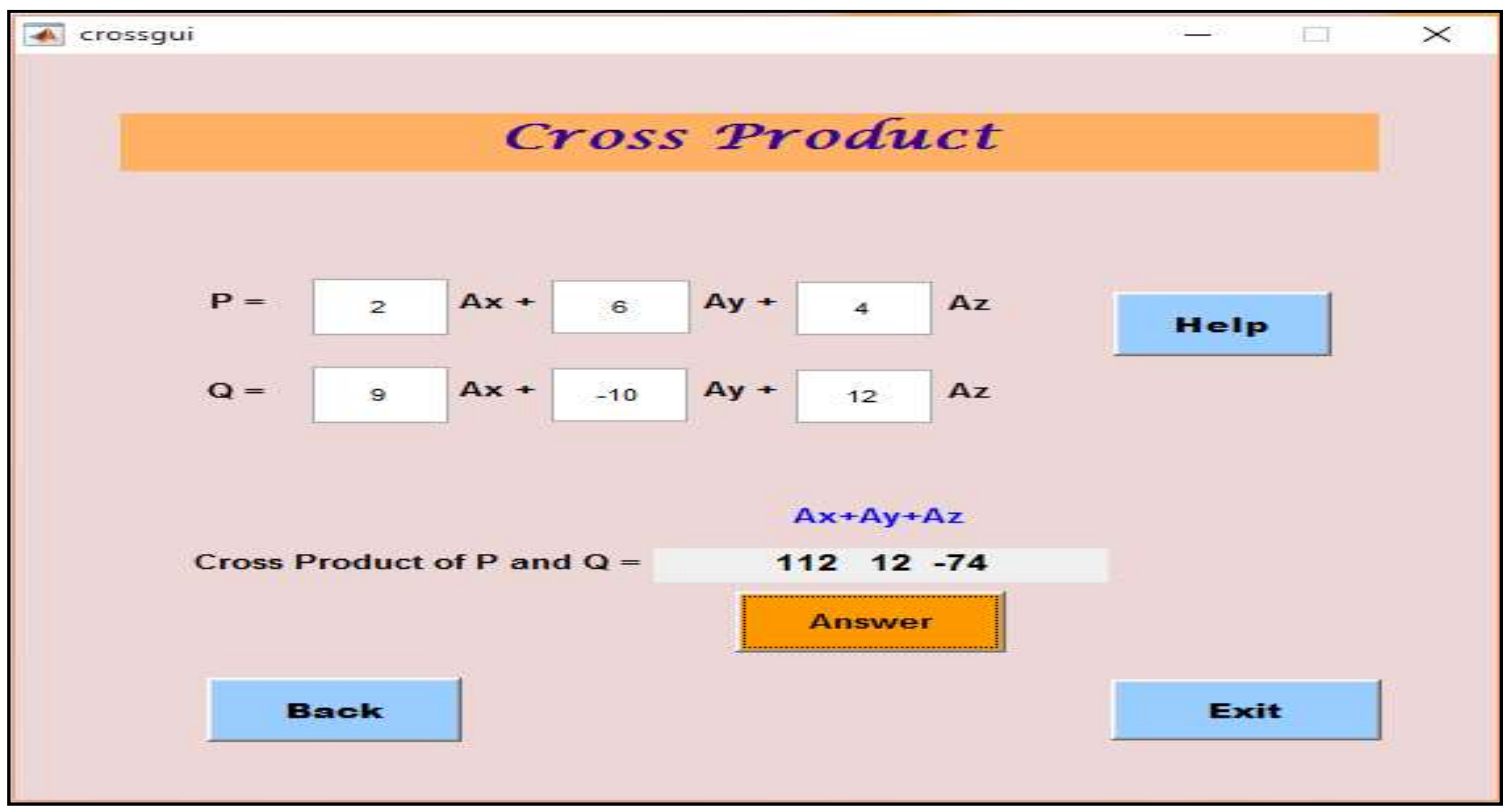

Fig.8. Cross Product calculator

Based on Fig. 9, the user need to identify which is the source point and the observation point. The source point is label as $Q$ usually will be given together with the charge value and the location of the point. Meanwhile, point of observation, $P$ is given the location of the point. The following values will be inserted and answer will be obtained as shown below. An aid is provided in the "Help" section. 


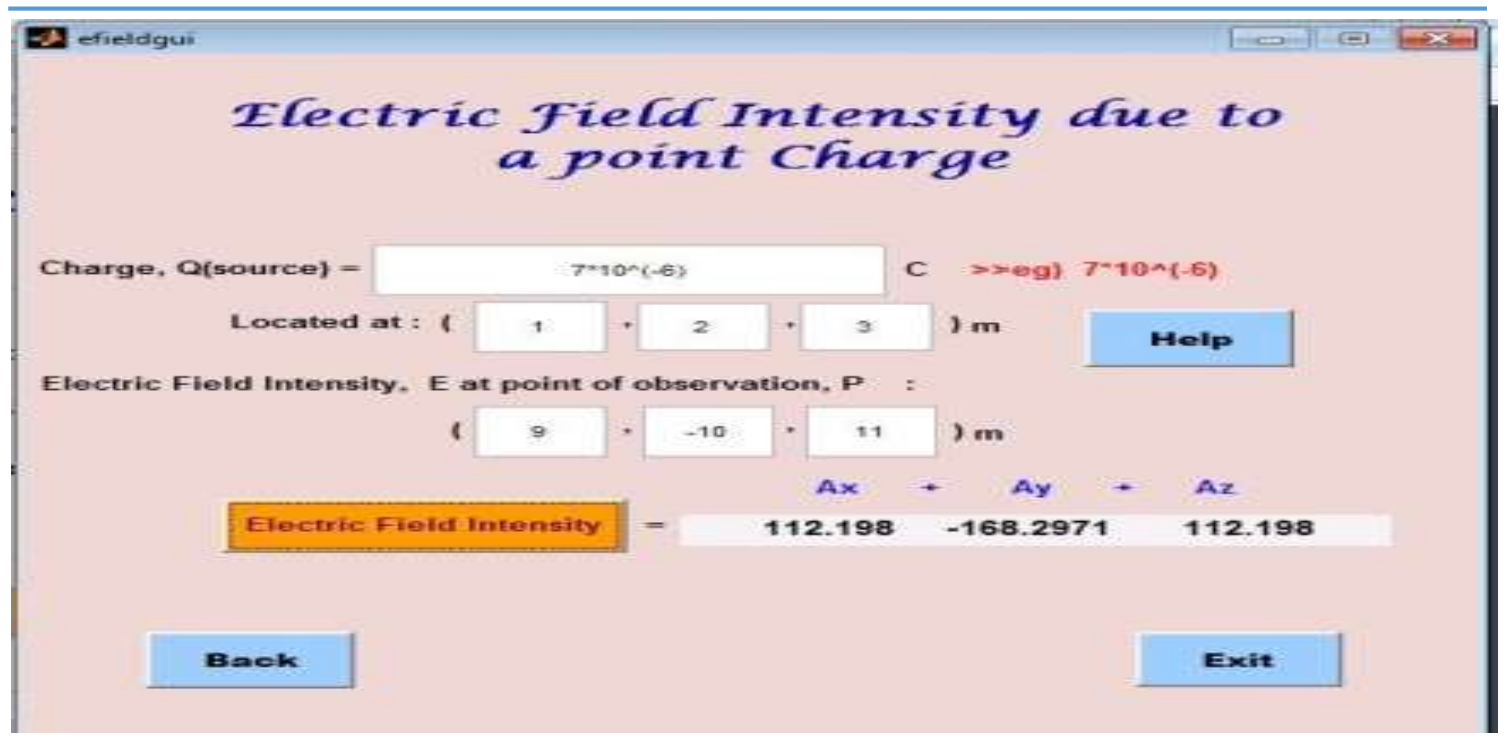

Fig.9. Intensity of electric field due to a point charge

This part is design with limitation up to 11 point charges of electric field that can be plotted. The range of axes can only be between the value of -5 to 5 . User can only enter the value as long as it is within the condition stated. The example is shown in Fig. 10.

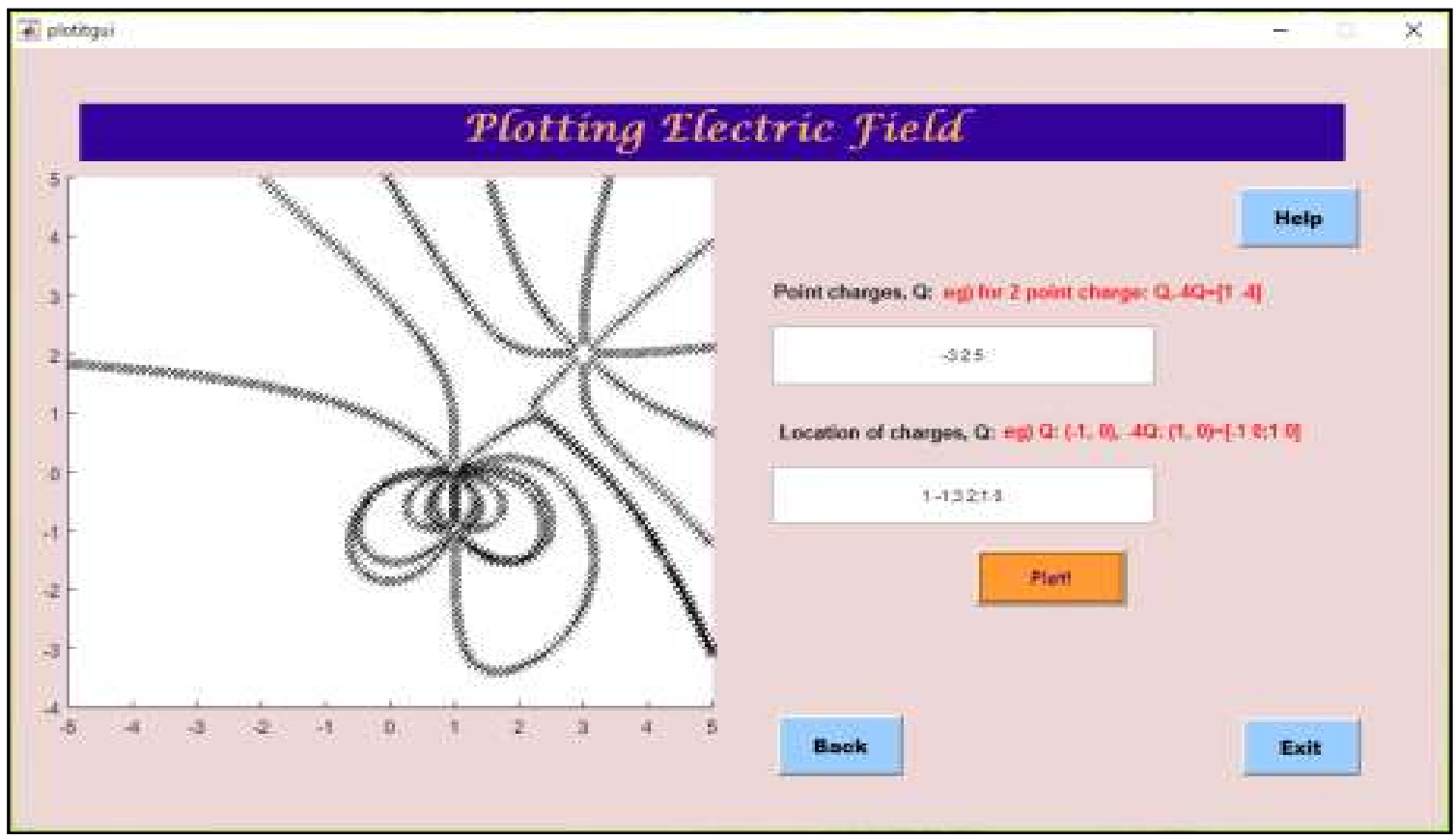

Fig.10. Example of electric field that have been plotted

This section can help user differentiate among free space wave propagation and good conductor wave propagation. There are three respective axes indicates electric wave, magnetic wave and power density as labelled in Fig. 11. User only needed to click onto the respective push button to see the wave propagation one at a time with duration about 10 seconds each. 


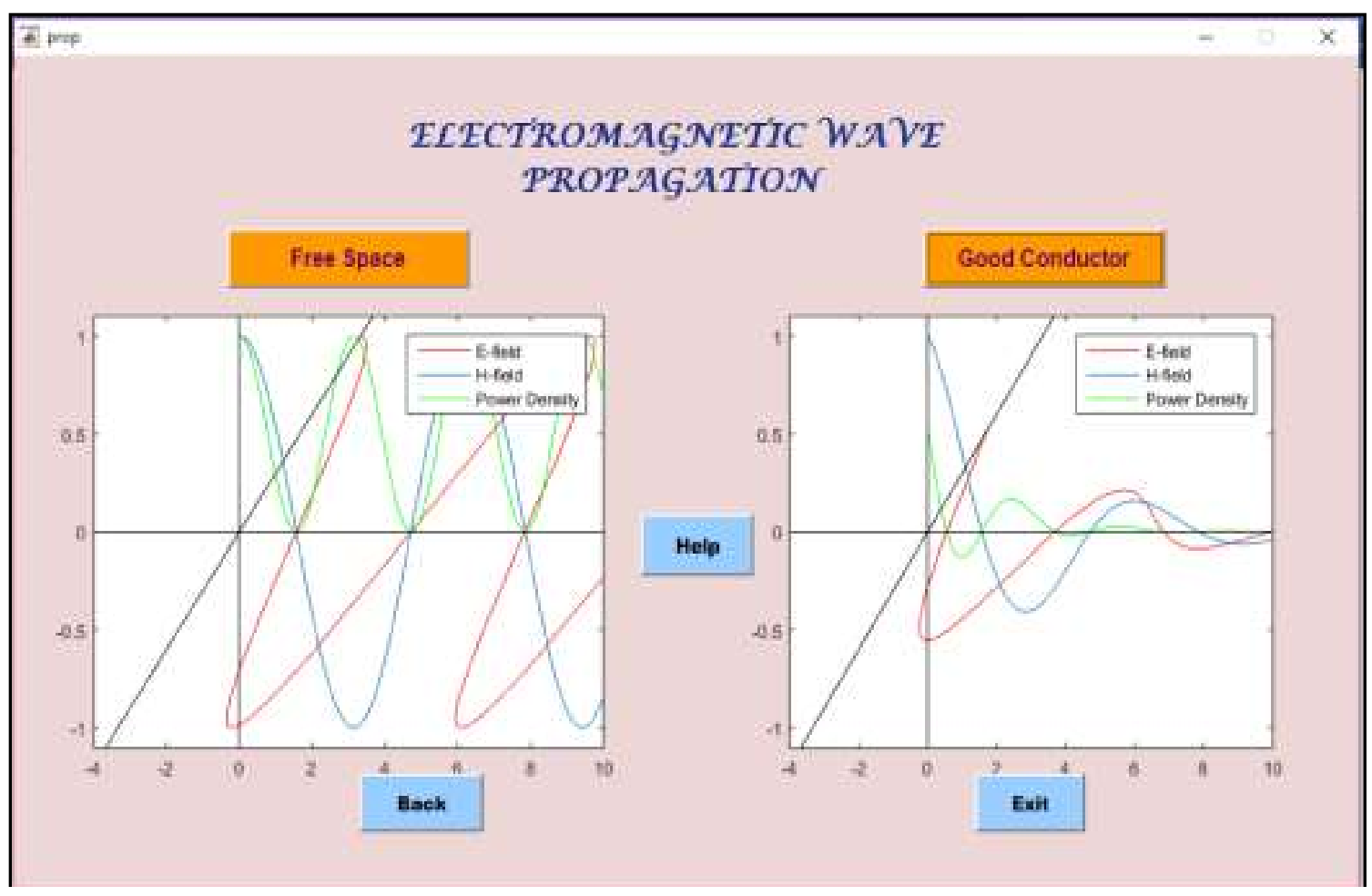

Fig. 11.Propagation of electromagnetic wave

There are three main types of coordinate system that has been stated before as depicted in Fig. 12. User must know which types of coordinate system that they have in order to convert it. For example, in Fig. 13 where the following data is in "Rectangular" dimension. The data is then inserted and the "Calculate" button is clicked. The result for the "Cylindrical" and "Spherical" dimension will appear in the appropriate boxes.

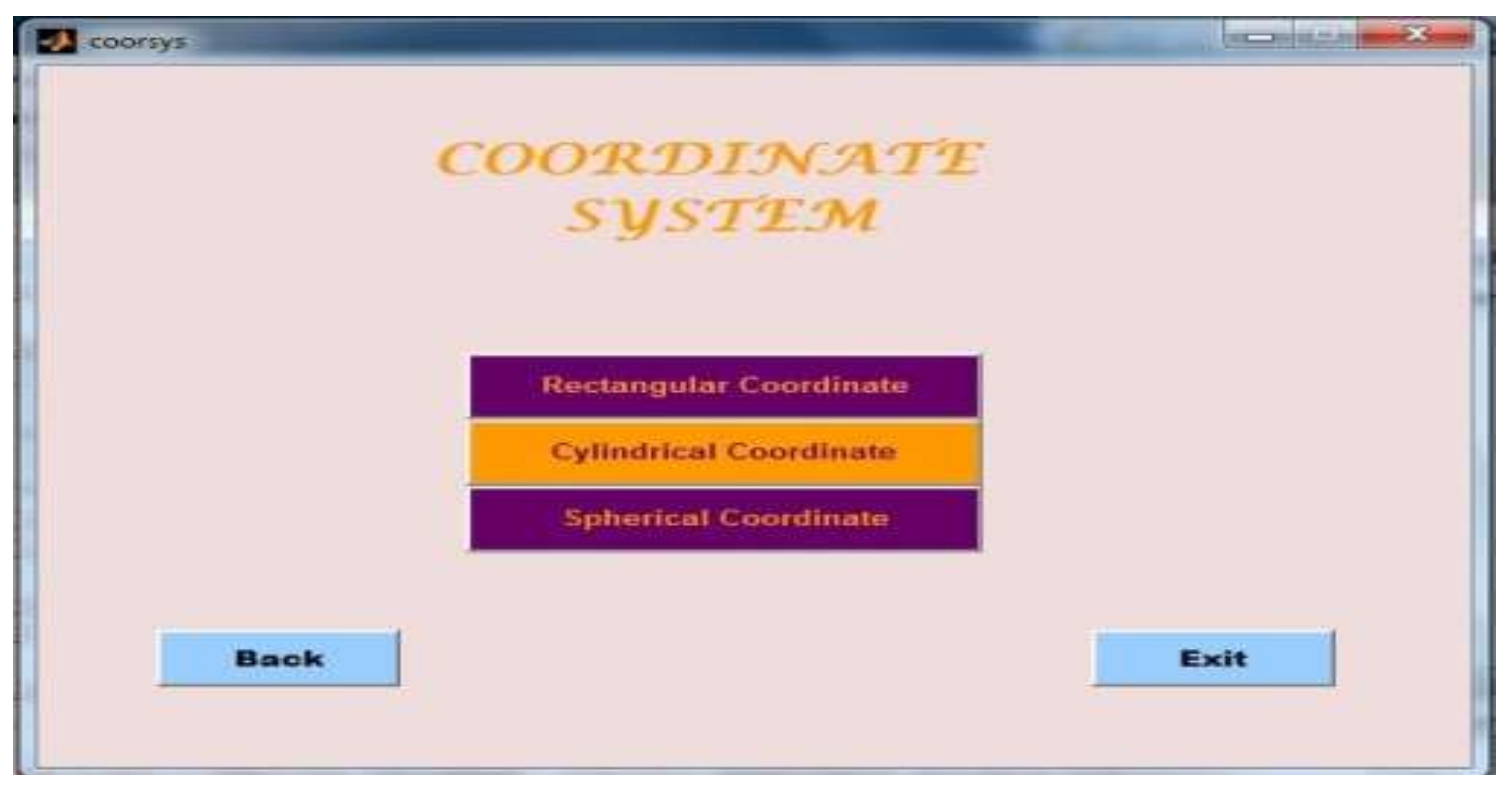

Fig.12. Coordinate system mode 


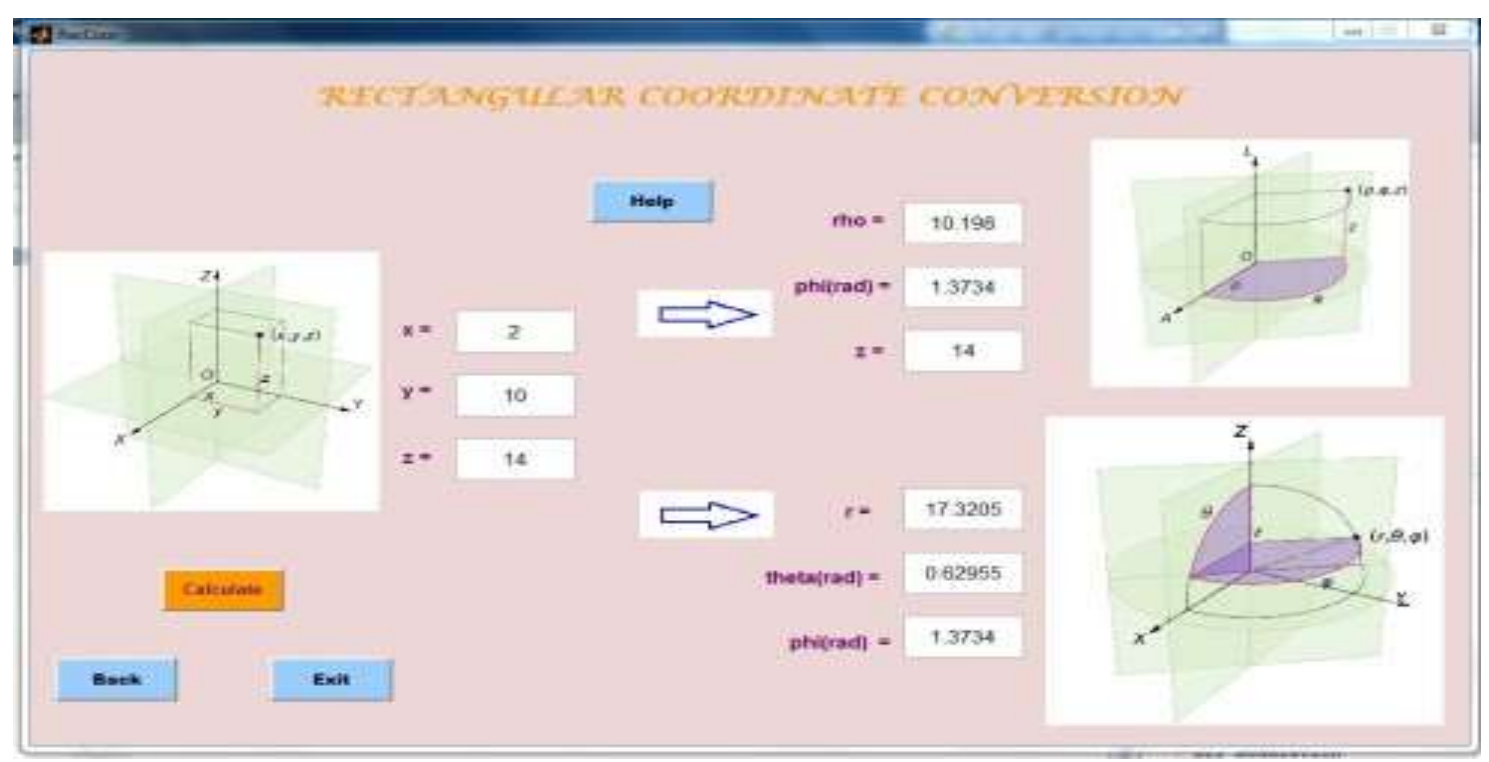

Fig.13. Coordinate conversion

In Amplitude Shift-Keying (ASK), user required to enter suitable values of carrier frequency and binary input of the signal. The signal will be plotted by clicking the "Generate!" button as in shown in Fig. 14. Same procedure goes to generating for QPSK (Fig. 15) and BPSK (Fig. 16). On the other hand, to plot the FSK signal the value required to be inserted is quite different from the other. Which are low signal frequency, high signal frequency, and binary input of the signal that needed to be inserted. The following is presented in Fig. 17. All frequencies parameters are in the form of Hertz $(\mathrm{Hz})$ unit. In All Modulation mode, we can see signals combination of the four-digital modulation. The appropriate parameters must be inserted and the plots will be generated. The example of the program is shown in Fig. 18.

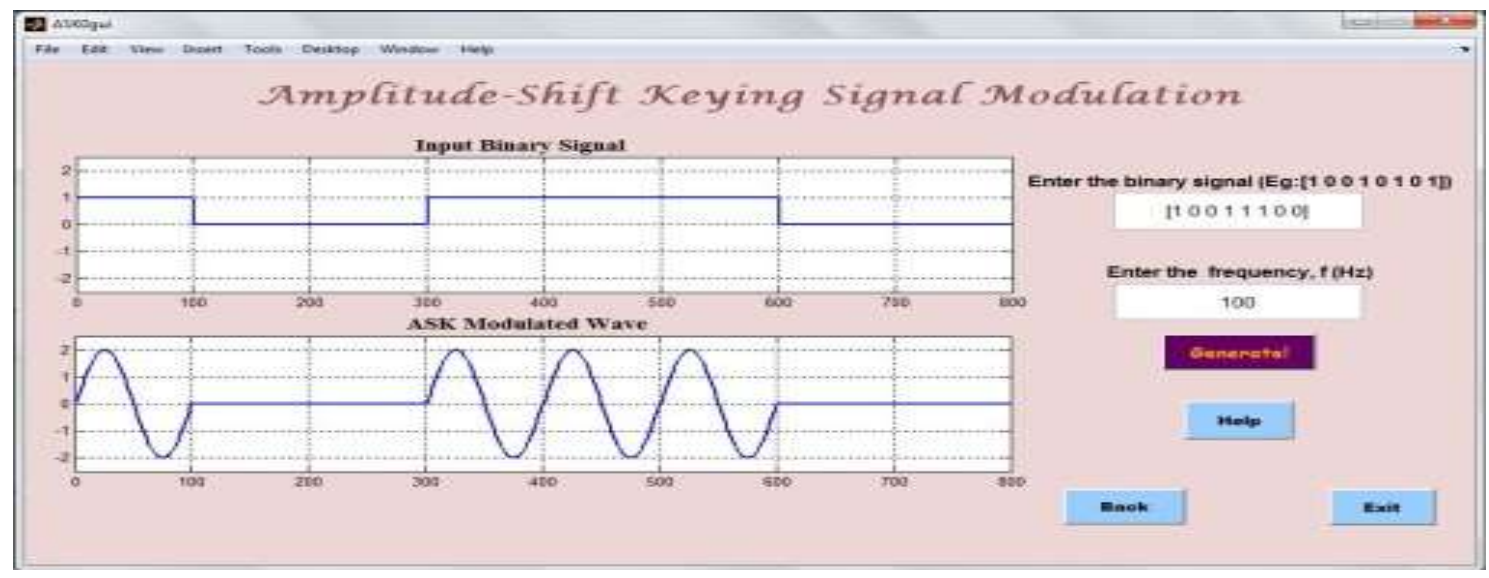

Fig.14. Amplitude-shift keying signal modulation 


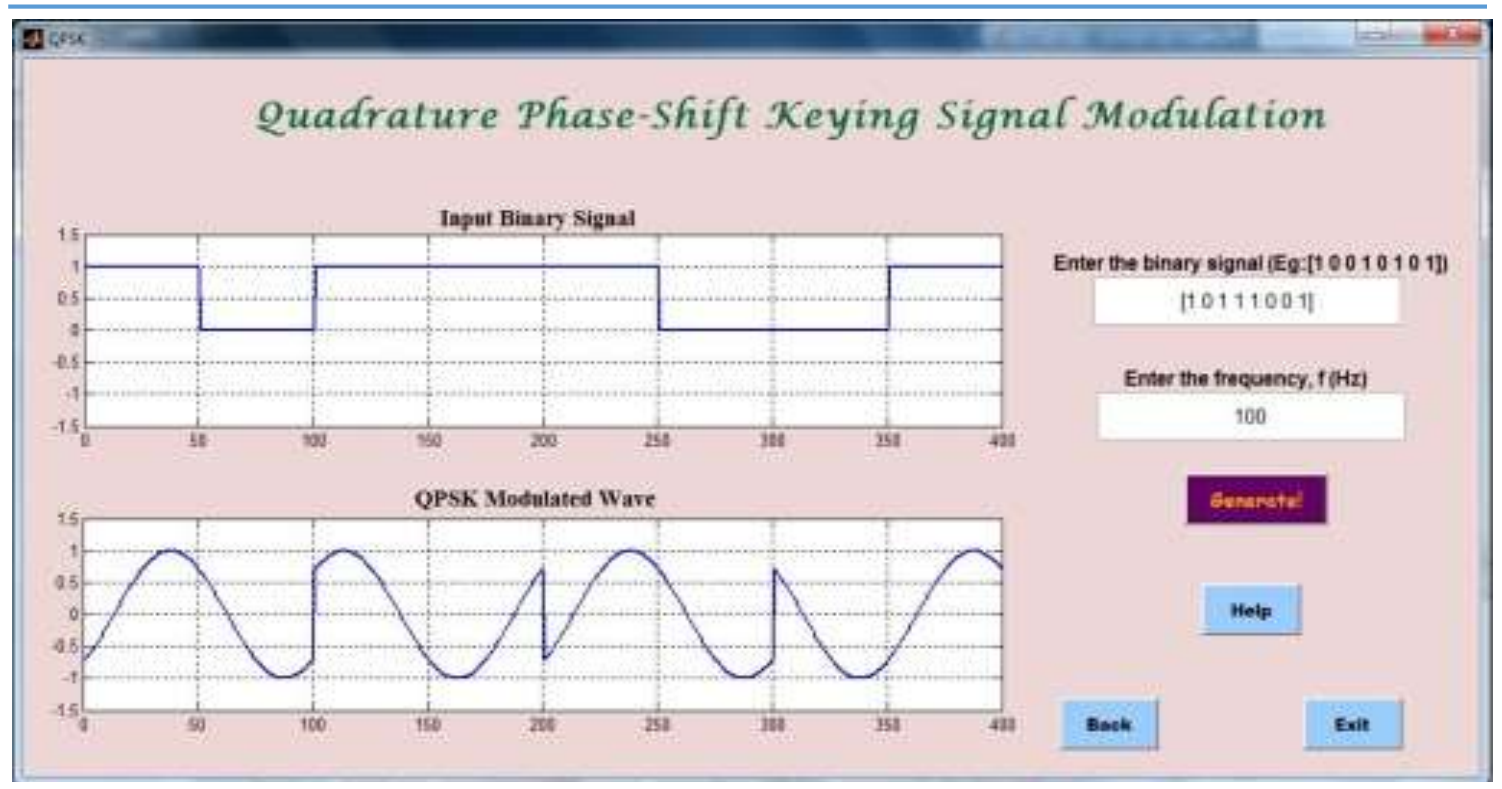

Fig.15. Quadrature phase-shift keying signal modulation

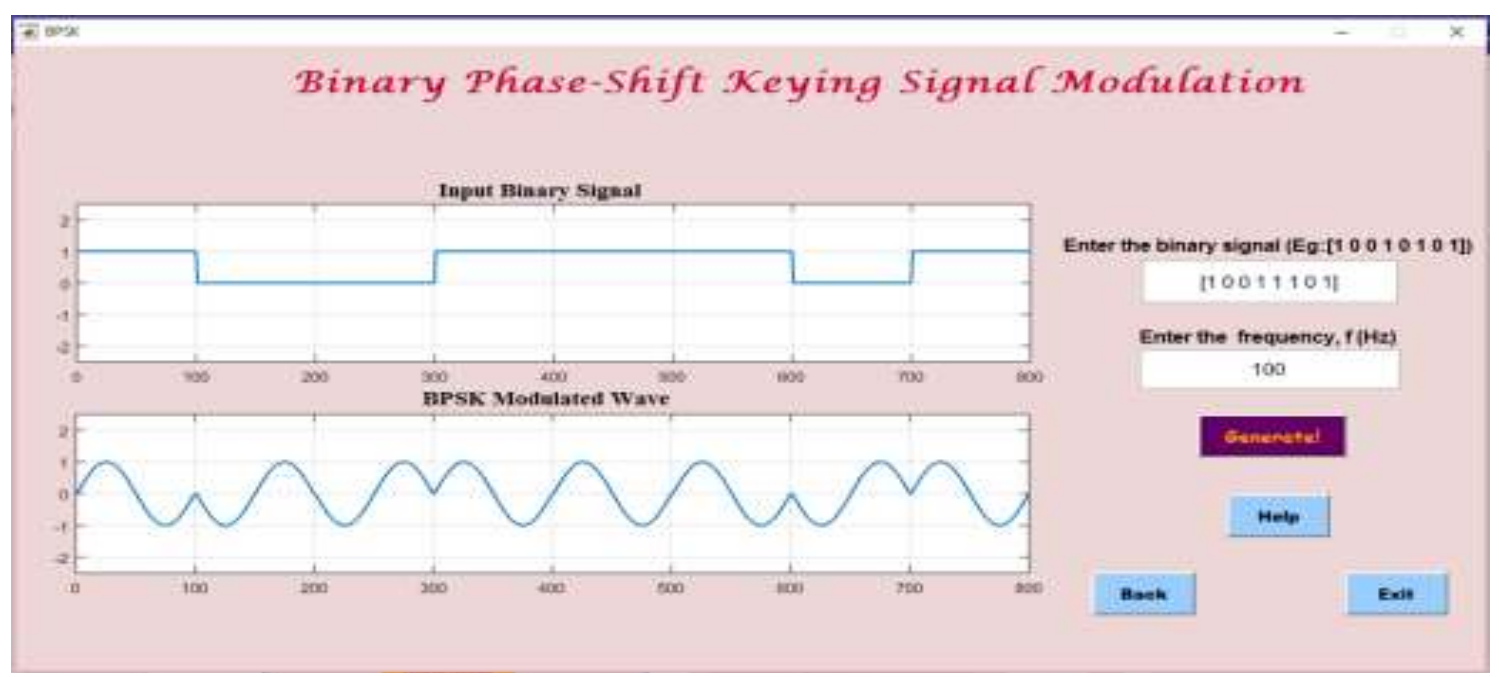

Fig.16. Binary phase-shift keying signal modulation

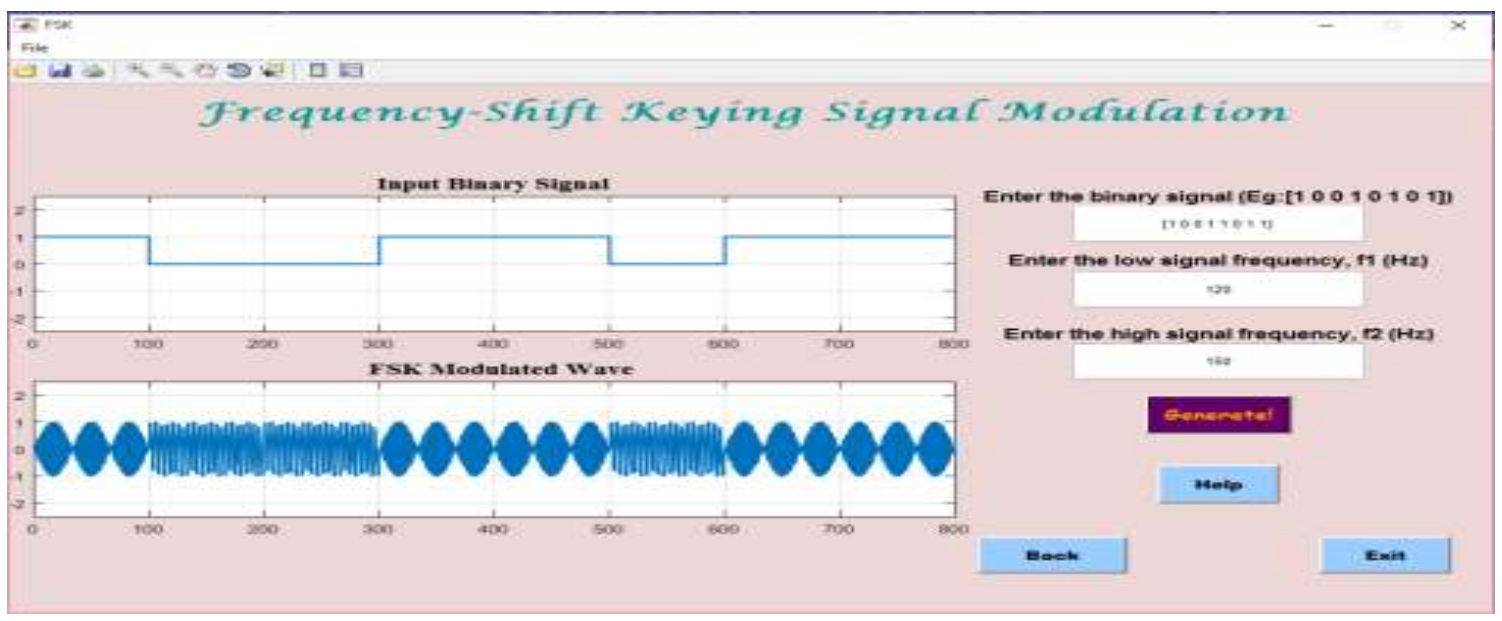

Fig.17. Frequency-shift keying signal modulation 


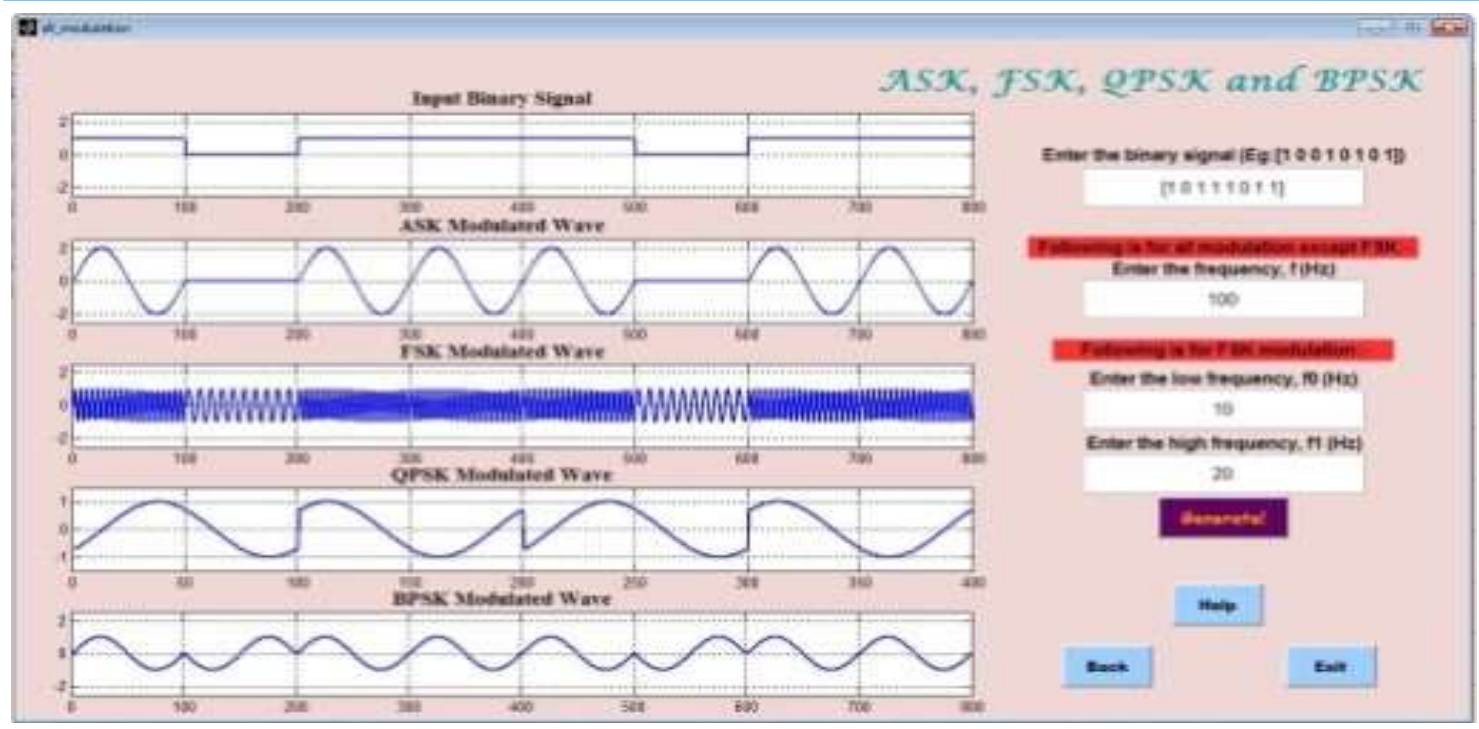

Fig.18. All signal modulation mode

High frequency analysis mode is designed with two options where user can choose to upload their own .wav file or choose it from the menu that had been listed. Then, user is required to follow the structure of the system from step 1 until 6 as shown in the GUI in Fig. 19.

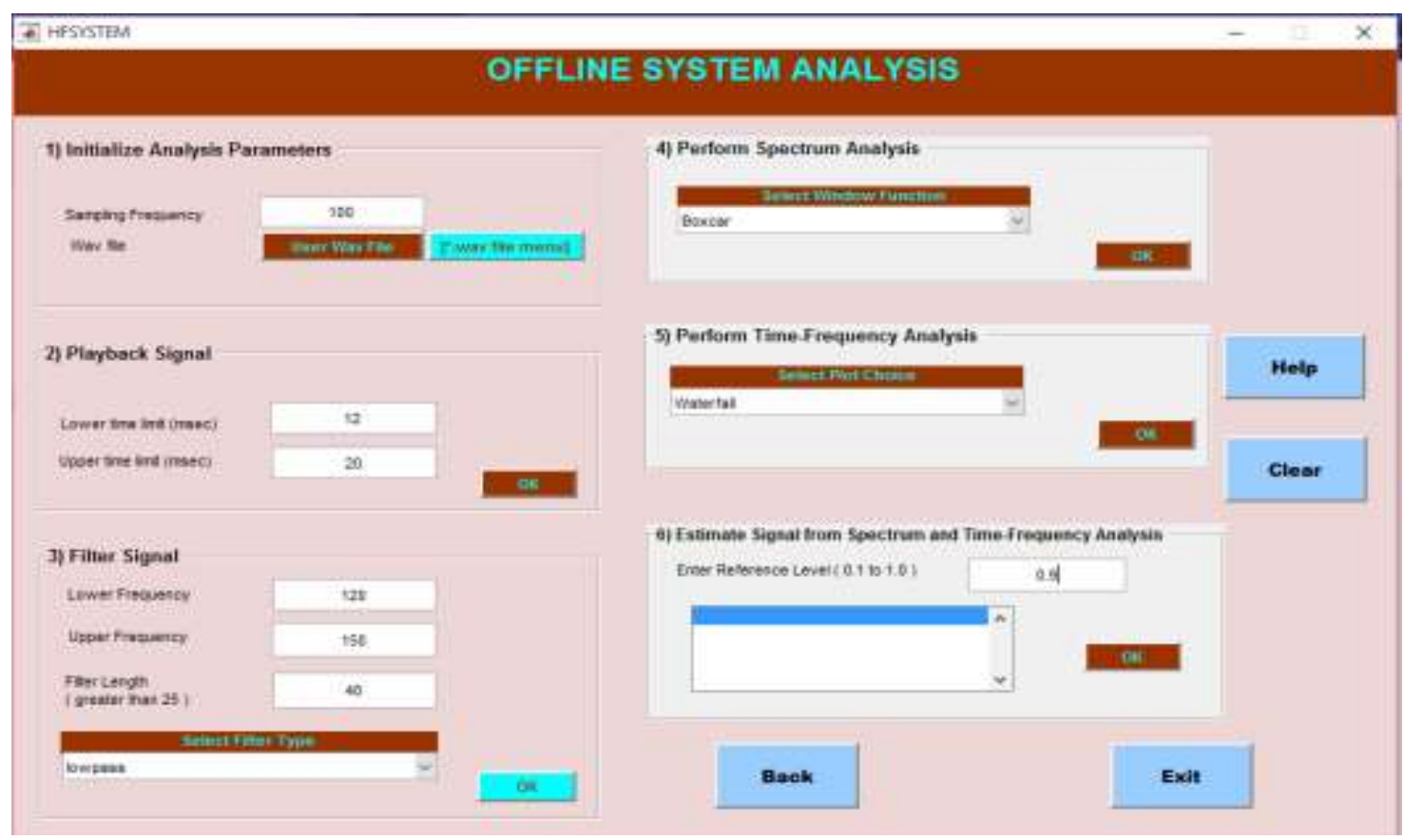

Fig.19. High frequency analysis

In this section, user can choose either "Star to Delta" or "Delta to Star" resistive conversion. The format is simple to understand. The user must identify the 3 phase circuit is in which form to be able to convert it. An appropriate value needs to be inserted and the user can click onto the "Calculate" button and see the results. Fig. 20 is the example of "Delta to Star" 
conversion.

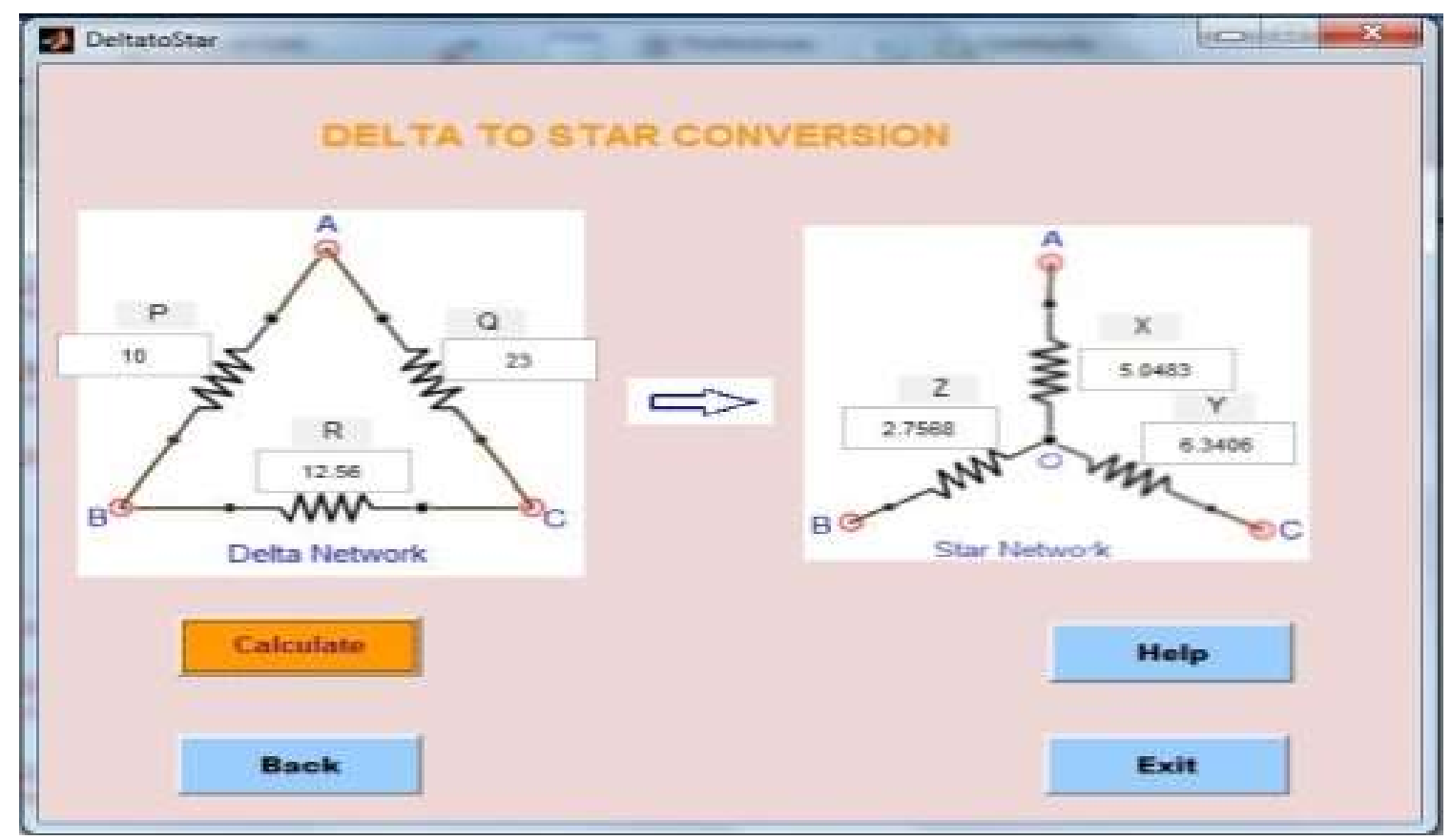

Fig.20. Delta to Star conversion mode

\section{CONCLUSION}

In conclusion, educational software using MATLAB and its GUI [14] has been developed which is appropriate for educational purposes either learning or teaching purposes. The system established is beneficial for the students to polish their understanding in the three subjects; electromagnetics, digital communication and power engineering. The standalone of this software is also developed. Thus, user does not need to install MATLAB [13] in order to use this educational software.

\section{ACKNOWLEDGEMENTS}

The author would like to acknowledge UniversitiTeknologi MARA (UiTM) for research funding under research grant 600-RMI/DANA 5/3/ARAS (15/2015). 


\section{REFERENCES}

[1] Lu L Y, Jin L Y.Design and implementation of linear control system teaching software using MATLAB. In 8th International Conference on Computer Science and Education, 2013, pp. $1393-1396$

[2] Karl J. A., Bjorn W. Computer-controlled systems: Theory and design.New York: Dover Publications, 2011

[3] Chris S., Toby B. Fundamentals of digital image processing: A practical approach with example in MATLAB.New Jersey: John Wiley and Sons, 2011

[4] Murthy S S, Bhuvaneswari G, Ahuja R K, Gao S. Analysis of self excited induction generator using MATLAB GUI methodology. In Joint International Conference on Power Electronics, Drives and Energy Systems and 2010 Power India, 2010, pp. 1-6

[5] Petropol S G D, Petropol S I,Campeanu A, Petrisor A. Using GUI of MATLAB to create a virtual laboratory to study an induction machine. In IEEE International Conference on "Computer as a Tool", 2007, pp. 2355-2360

[6] Sadiku M. N. O. Elements of electromagnetics. England: Oxford University Press, 2014

[7] Joseph E.Schaum's outline of electromagnetics.New York: McGraw Hill, 2010

[8] Uday A. B. Electromagnetic wave theory.Pune: Technical Publications, 2009

[9] Gerd M., Matthias S. Electromagnetic field theory: A collection of problem.Berlin: Springer Science and Business Media, 2012

[10]Naim N F, Nordin A H, Rahman H A. Interactive learning software forcommunication engineering subject.In IEEE International Conference on System Engineering and Technology, 2011, pp. 225-230

[11]Chitode J. S. Digital communication.Pune: Technical Publications, 2011

[12]Zlatan S. Computer aided design in power engineering: Application of software tools. Berlin: Springer Science and Business Media, 2012

[13]Nawi B, Sulaini B, Mohd Z A R, Shamsul A Z,Zairi I R. PID voltage control for DC motor using MATLAB Simulink and Arduino microcontroller. Journal of Applied Environmental and Biological Sciences, 2015, 5(9):166-173 
[14]Mohamad T M, Zairi I R, Wan A K W C, Fadhli D H M F.Fitness cycling device with graphical user interface based on IEEE 802.15.4 transceiver for real time monitoring.Journal of Applied Environmental and Biological Sciences, 2014, 4(12):108-114

\section{How to cite this article:}

Naim N F, Zaini H I M, Sarnin S S, Ya'acob N. Interactive learning software for electrical engineering subjects using matlab and its gui. J. Fundam. Appl. Sci., 2017, 9(5S), 260-276. 